\title{
ÜBER DIE EINDEUTIGEN LÖSUNGEN EINER HOMOGENEN ALGEBRAISCHEN DIFFERENTIALGLEICHUNG ZWEITER ORDNUNG
}

\author{
NORBERT STEINMETZ
}

\section{Einleitung}

Es sei $\Omega\left(z, w, w_{1}, w_{2}\right)$ ein nichttriviales homogenes Polynom in den Veränderlichen $w, w_{1}$ und $w_{2}$ mit rationalen Koeffizienten. In [7] wurde gezeigt, daß sämtliche in $\boldsymbol{C}$ meromorphen Lösungen der algebraischen Differentialgleichung

$$
\Omega\left(z, w, w^{\prime}, w^{\prime \prime}\right)=0
$$

sich in der Form

$$
w(z)=\frac{g_{1}(z)}{g_{2}(z)} \exp g_{3}(z)
$$

mit ganzen Funktionen $g_{j}$ von endlicher Ordnung darstellen lassen. Dabei wurde außer acht gelassen, ob und unter welchen Voraussetzungen (1) solche Lösungen zuläßt. In diesem Zusammenhang stellt sich die Frage nach möglichst schwachen Bedingungen dafür, daß jede Lösung von (1) in $C$ meromorph ist, daß also die analytische Fortsetzung eines beliebigen Lösungselements bis auf isolierte Polstellen unbeschränkt durchführbar ist. Um solche Bedingungen aufzufinden, ist es sehr hilfreich, die notwendigen Bedingungen zu kennen, die sich aus der Existenz wenigstens einer in $|z|<\infty$ eindeutigen Lösung von (1) ergeben. Es zeigt sich zumindest in den hier behandelten Fällen, daß die "Struktur" der Gleichung (1), d. h. die Abhängigkeit von $\Omega$ von den Variablen $w, w_{1}$ und $w_{2}$, nur durch die notwendigen Bedingungen bestimmt wird, während Voraussetzungen nur an die auftretenden Koeffizienten schließlich garantieren, da $ß$ jede Lösung zu einer in $C$ meromorphen Funktion fortgesetzt werden kann.

Diese Arbeit beschränkt sich auf Gleichungstypen (1), in denen $w^{\prime \prime}$ linear oder quadratisch auftritt, also

$$
M\left(z, w, w^{\prime}\right) w^{\prime \prime}+N\left(z, w, w^{\prime}\right)=0
$$

und

$$
L\left(z, w, w^{\prime}\right)\left(w^{\prime \prime}\right)^{2}+2 M\left(z, w, w^{\prime}\right) w^{\prime \prime}+N\left(z, w, w^{\prime}\right)=0,
$$


wobei lineare Gleichungen $w^{\prime \prime}+a(z) w^{\prime}+b(z) w=0$ außer acht gelassen werden können. Es wird stets angenommen, ohne daß dies jedesmal wiederholt wird, daß (I) bzw. (II) irreduzibel sind, d.h. $\Omega$ soll nicht als Produkt zweier homogener Polynome $\Omega_{1}$ und $\Omega_{2}$ darstellbar sein. Dies bedeutet keine Einschränkung, da man ansonsten die Gleichung $\Omega_{1}=0$ oder $\Omega_{2}=0$ anstelle von (1) betrachten würde. Eine Einschränkung bedeutet hingegen die Voraussetzung

(E) Die Differentialgleichung (I) bzw. (II) besitzt eine in $|z|<\infty$ meromorphe Lösung mit nichtrationaler logarithmischer Ableitung $w^{\prime} / w$

zur Gewinnung notwendiger Bedingungen. Andererseits sind aber ohne diese zusätzliche Voraussetzung an $w^{\prime} / w$ überhaupt keine sinnvollen Aussagen möglich. Man denke etwa an die Exponentialfunktion $w=e^{z}$, die jeder homogenen Gleichung $\Omega\left(z, w, w^{\prime}, w^{\prime \prime}\right)=0$ mit $\Omega(z, 1,1,1) \equiv 0$ genügt. Ähnliche Beispiele findet man für jede Funktion $w=\left(P_{1}(z) / P_{2}(z)\right) e^{P_{3}(z)}, P_{j}$ Polynome, mit rationaler logarithmischer Ableitung.

Ein wesentliches Hilfsmittel für die folgenden Betrachtungen bildet die Nevanlinnasche Theorie der meromorphen Funktionen. Es werden die üblichen Bezeichnungen verwendet, insbesondere die Abkürzung $S(r, w)$ für jede Funktion, die der Bedingung $S(r, w)=o(T(r, w))$ für $r \rightarrow+\infty$ außerhalb eventuell einer Menge von endlichem Maß genügt (vgl. [2], [4]). $T(r, w)$ ist die charakteristische Funktion von $w$.

\section{Notwendige Bedingungen}

Diese sind sehr einfach im Falle der Gleichung (I).

Satz 1. Unter der Voraussetzung (E) hat (I) notwendig die Gestalt

$$
w w^{\prime \prime}+a(z) w^{2}+b(z) w w^{\prime}+c(z) w^{2}=0 .
$$

Dabei sind $a, b$ und $c$ rationale Funktionen, und es ist $c(z) \not \equiv 0$.

Bemerkung. Der Fall $c(z) \equiv 0$ bedeutet, daß eine lineare Differentialgleichung vorliegt, und diese sollen ja ausgeschlossen sein.

Es kann mehr ausgesagt werden, wenn alle Koeffizienten in (3) Polynome sind. Diese Voraussetzung ist nicht sehr einschränkend, da die Polstellen der Koeffizienten i.a. feste singuläre Stellen der Lösungen sind.

Als Beispiel dient die Gleichung

$$
w w^{\prime \prime}+\left(\frac{1}{z^{2}}-1\right) w^{2}+\left(\frac{1}{z^{2}}-2\right) w^{2}=0
$$

Jede von $C e^{ \pm i z}$ verschiedene Lösung ist bei $z=0$ wesentlich singulär. Dies folgt aus dem Verhalten der logarithmischen Ableitung $w^{\prime} / w=y=\tan (z+1 / z+\gamma)$, die der Riccatischen Differentialgleichung $y^{\prime}=\left(1-1 / z^{2}\right)\left(1+y^{2}\right)$ genügt. 
Satz 2. Sind die Koeffizienten in (3) Polynome und ist (E) erfüllt, so gilt entweder (a) $c(z) \equiv-1$ und jede Lösung ist eine ganze Funktion der Gestalt $w(z)=\exp g(z)$, wobei $y=g^{\prime}$ der linearen Differentialgleichung $y^{\prime}+a(z)+b(z) y=0$ genügt, oder (b) $c(z) \equiv-1+1 / n$, wobei $n \neq 0,1$ eine ganze Zahl ist. Jede Lösung hat die Gestalt $w=v^{n}$, wobei $v$ die lineare Differentialgleichung $v^{\prime \prime}+b(z) v^{\prime}+(a(z) / n) v=0$ löst.

Bemerkung. Die Integration der Gleichung (3) läßt sich also stets auf die Integration einer linearen Differentialgleichung zurückführen.

Ein dem Satz 1 entsprechendes Ergebnis gilt auch für Gleichung (II).

Satz 3. Unter der Voraussetzung (E) hat (II) notwendigerweise die Gestalt

$$
\left(w w^{\prime \prime}\right)^{2}+2 P\left(z, w, w^{\prime}\right) w w^{\prime \prime}+Q\left(z, w, w^{\prime}\right)=0 .
$$

Dabei ist

$$
P\left(z, w, w^{\prime}\right)=\sum_{j=0}^{2} a_{j}(z) w^{2-j}\left(w^{\prime}\right)^{j}
$$

und

$$
Q\left(z, w, w^{\prime}\right)=\sum_{j=0}^{4} b_{j}(z) w^{4-j}\left(w^{\prime}\right)^{j}
$$

die Koeffzienten $a_{j}, b_{j}$ sind rationale Funktionen.

Bemerkung. Eventuell ist ein allen Polynomen $L, M$ und $N$ gemeinsamer, nur von $z$ abhängiger Faktor gekürzt worden. Es ist auch möglich, daß in (4) ein Faktor $w$ oder $w^{2}$ wegfällt (falls $b_{4} \equiv 0$ oder sogar $b_{4} \equiv b_{3} \equiv a_{2} \equiv 0$ ist).

Auch im Falle der Gleichung (4) sind weitergehende Aussagen möglich, wenn die auftretenden Koeffizienten Polynome sind. Um die Ergebnisse nicht zu unübersichtlich erscheinen zu lassen, wird eine Fallunterscheidung entsprechend der Zahl der Null- und Polstellen von $w$ getroffen.

\section{Lösungen mit nur endlich vielen Null- und Polstellen}

In diesem Abschnitt wird generell angenommen, daß die in Bedingung (E) vorkommende Lösung nur endlich viele Null- und Polstellen besitzt (und damit notwendigerweise unendliche Ordnung hat). Es sei

$$
D(z, y):=\left(a_{2}^{2}-b_{4}\right) y^{4}+\left(2 a_{1} a_{2}-b_{3}\right) y^{3}+\left(a_{1}^{2}+2 a_{0} a_{2}-b_{2}\right) y^{2}+\left(2 a_{0} a_{1}-b_{1}\right) y+a_{0}^{2}-b_{0}
$$

die Diskriminante der Gleichung (4).

Satz 4. Es gelte (E), und die Koeffizienten in (4) seien Polynome. Dann ist

$$
a_{2}(z) \equiv-1, \quad b_{4}(z) \equiv 1,
$$


und es gibt ein Polynom $p(z)$ und eine Konstante $x \neq 0$, so daß sich $D(z, y)$ in der Form

$$
D(z, y)=c(z)(y-p(z)), \quad c:=2 a_{0} a_{1}-b_{1} \not \equiv 0
$$

oder

$$
D(z, y)=c(z)\left[(y-p(z))^{2}-x^{2}\right], \quad c:=a_{1}^{2}+2 a_{0} a_{2}-b_{2} \not \equiv 0
$$

darstellen läßt. Die Funktion $p(z)$ bzw. $p(z) \pm x$ ist Lösung der Differentialgleichung $y^{\prime}+a_{0}+a_{1} y=0$, und im Falle (b) ist zusätzlich $a_{1}(z) \equiv 0$.

Bemerkung. Die Substitution

$$
w=u \exp \left[\int_{0}^{z} p(z) d z\right]
$$

führt in den nach Satz 4 einzig möglichen Fällen auf die Gleichungen

$$
\left[\left(\frac{u^{\prime}}{u}\right)^{\prime}+a_{1}(z) \frac{u^{\prime}}{u}\right]^{2}=c(z) \frac{u^{\prime}}{u}
$$

bzw.

$$
\left[\left(\frac{u^{\prime}}{u}\right)^{\prime}\right]^{2}=c(z)\left[\left(\frac{u^{\prime}}{u}\right)^{2}-x^{2}\right]
$$

Es genügt daher, diese Gleichungen zu untersuchen.

Bei beliebigem $c(z)$ ist nicht $\mathrm{zu}$ erwarten, daß alle Lösungen eindeutig sind. So hat die allgemeine Lösung von $\left[\left(u^{\prime} / u\right)^{\prime}+u^{\prime} / u\right]^{2}=z\left(u^{\prime} / u\right)$ bei $z=0$ einen algebraischen Verzweigungspunkt. Dagegen gilt

Satz 5. Ist $c(z)=\gamma^{2}(z), \gamma$ ein Polynom, so ist jede Lösung u(z) von (9) bzw. (10) eine ganze Funktion ohne Nullstellen.

In dem Beispiel ist gerade die Zusatzbedingung für $c(z)=z$ verletzt.

\section{Lösungen mit unendlich vielen Null- oder Polstellen}

Die Null- und Polstellen $z_{1}, z_{2}, \ldots$ von $w$ sind einfache Pole von $w^{\prime} / w$ mit ganzzahligen Residuen $R_{1}, R_{2}, \ldots$ Aus der Differentialgleichung (17) für $y=w^{\prime} / w$ (vgl. Abschnitt 6) erhält man für $z=z_{j}$ und $R=R_{j}$ die quadratische Gleichung

$$
1-2\left(1+a_{2}(z)\right) R+\left(1+2 a_{2}(z)+b_{4}(z)\right) R^{2}=0 .
$$

Da die Lösungen von (4) bewegliche Null- oder Polstellen besitzen, bedeutet die folgende Residuenbedingung keine wesentliche Einschränkung:

(R) Für jedes $z \in \boldsymbol{C}$ besitzt Gleichung (11) nur ganzzahlige Lösungen.

Satz 6. In der Differentialgleichung (4) seien die Koeffizienten Polynome. Weiter seien die Voraussetzungen $(E)$ und $(R)$ erfüllt. Dann sind $a_{2}(z)$ und $b_{4}(z)$ konstant, und 
es liegt genau eine der folgenden Gleichungen vor:

(a)

$$
\left[\left(\frac{w^{\prime}}{w}\right)^{\prime}-p^{\prime}+\frac{1}{n}\left(\left(\frac{w^{\prime}}{w}-p\right)^{2}-\varkappa^{2}\right)\right]^{2}=c(z)\left[\left(\frac{w^{\prime}}{w}-p\right)^{2}-\varkappa^{2}\right]
$$

(b)

$$
\left[\left(\frac{w^{\prime}}{w}\right)^{\prime}-p^{\prime}+\frac{1}{n}\left(\frac{w^{\prime}}{w}-p\right)^{2}\right]^{2}=c(z)\left[\left(\frac{w^{\prime}}{w}-p\right)\left(z-z_{1}\right)-n\right]\left(\frac{w^{\prime}}{w}-p\right) ;
$$

(c)

$$
\left[\left(\frac{w^{\prime}}{w}\right)^{\prime}-p^{\prime}+\frac{1}{n}\left(\frac{w^{\prime}}{w}-p\right)^{2}\right]^{2}=c(z)\left[\left(\frac{w^{\prime}}{w}-p\right)\left(z-z_{1}\right)-n\right]\left[\left(\frac{w^{\prime}}{w}-p\right)\left(z-z_{2}\right)-n\right]
$$

(d)

$$
\left[\left(\frac{w^{\prime}}{w}\right)^{\prime}-p^{\prime}\right]^{2}=\frac{1}{n^{2}} \prod_{j=1}^{4}\left(\frac{w^{\prime}}{w}-p-\varkappa_{j}\right)
$$

(e)

$$
\left[\left(\frac{w^{\prime}}{w}\right)^{\prime}-p^{\prime}+\frac{1}{2 n}\left[\left(\frac{w^{\prime}}{w}-p\right)^{2}-\chi^{2}\right)\right]^{2}=\frac{1}{4 n^{2}}\left[\left(\frac{w^{\prime}}{w}-p\right)^{2}-\chi^{2}\right]\left[\frac{w^{\prime}}{w}-p-s\right]^{2}
$$

(f)

$$
\left[\left(\frac{w^{\prime}}{w}\right)^{\prime}-p^{\prime}+\frac{n+m}{2 n m}\left(\left(\frac{w^{\prime}}{w}-p\right)^{2}-\varkappa^{2}\right)\right]^{2}=\left(\frac{n-m}{2 n m}\right)^{2}\left[\left(\frac{w^{\prime}}{w}-p\right)^{2}-\varkappa^{2}\right]\left[\frac{w^{\prime}}{w}-p-s\right]^{2} .
$$

Dabei sind $c, p$ und $s$ Polynome $(c(z) \neq 0), n$ und $m(n \neq m)$ ganze und $z_{1}, z_{2}, \varkappa_{,} \varkappa_{1}, \varkappa_{2}$, $\varkappa_{3}, \varkappa_{4}$ komplexe Zahlen $\left(z_{1} \neq z_{2}, x \neq 0, x_{i} \neq \varkappa_{j}\right.$ für $\left.i \neq j\right)$.

Die notwendigen Bedingungen von Satz 6 sind "fast" hinreichend. Genauer gilt

Satz 7. Die Lösungen der Gleichungen (d), (e) und (f) aus Satz 6 und - falls $c(z) \equiv \gamma^{2}(z), \gamma$ ein Polynom, gilt - auch von (a), (b) und (c) sind in C meromorphe Funktionen.

Bemerkung. Neben den Lösungen $w=\exp \left[\int^{z} p(z) d z \pm x z\right]$ von (a), (e) und (f), $w=\exp \left[\int^{z} p(z) d z+\varkappa_{j} z\right]$ von (d), $w=\exp \left[\int^{z} p(z) d z\right]$ von (b) und $w=\left(z-z_{j}\right)^{n} \cdot$ $\exp \left[\int^{z} p(z) d z\right]$ von (b) und (c) haben alle Lösungen unendlich viele Null- oder Polstellen.

\section{Beweis der Sätze 1, 2 und 3}

Die Beweise der Sätze 1 und 2 verlaufen zum größten Teil parallel. Nach Voraussetzung ist

$$
y:=w^{\prime} / w,
$$

wobei $w$ die nach (E) vorhandene Lösung von (I) bzw. (II) ist, eine in $C$ transzendente Funktion. Mit Hilfe von

$$
y^{\prime}+y^{2}=w^{\prime \prime} / w
$$


und der Homogenität der Gleichungen (I) bzw. (II) erhält man für $y$

$$
M(z, 1, y)\left(y^{\prime}+y^{2}\right)+N(z, 1, y)=0
$$

bzw.

$$
A(z, y)+2 B(z, y) y^{\prime}+C(z, y) y^{\prime 2}=0
$$

mit

und

$$
\begin{aligned}
& A(z, y):=L(z, 1, y) y^{4}+2 M(z, 1, y) y^{2}+N(z, 1, y) \\
& B(z, y):=L(z, 1, y) y^{2}+M(z, 1, y)
\end{aligned}
$$

$$
C(z, y):=L(z, 1, y) \text {. }
$$

Die Gleichungen (14), (15) sind mit (I), (II) irreduzibel. Nach dem Satz von Malmquist [3] ist (14) notwendigerweise eine Riccatische Differentialgleichung

$$
y^{\prime}+a(z)+b(z) y+(c(z)+1) y^{2}=0 .
$$

Hieraus folgt bereits die Behauptung von Satz 1, wenn die Substitution (12) rückgängig gemacht wird.

Zum vollständigen Beweis von Satz 3 wird ein dem Malmquistschen Satz analoges Ergebnis benötigt. Da es auch an anderer Stelle benutzt wird, sei es hier als Hilfssatz formuliert.

Hilfssatz. Für die Existenz einer in C transzendenten meromorphen Lösung der irreduziblen Gleichung (15) müssen folgende Bedingungen erfüllt sein:

$1^{\circ} C(z, y)$ ist unabhängig von $y$ (so daß o.B.d.A. $C(z, y) \equiv 1$ angenommen werden kann).

$2^{\circ} A(z, y)$ bzw. B(z,y) haben in bezug auf $y$ den Grad höchstens 4 bzw. 2.

$3^{\circ}$ Die Gleichung $D(z, y):=B^{2}(z, y)-A(z, y) C(z, y) \equiv 0$ besitzt wenigstens eine Wurzel $y=\varrho(z)$ von ungerader Vielfachheit.

$4^{\circ}$ Jede Wurzel der Gleichung $D(z, y) \equiv 0$ von ungerader Vielfachheit genügt auch der Riccatischen Differentialgleichung $y^{\prime}+B(z, y)=0$.

Bemerkung. Beweise für $1^{\circ}, 2^{\circ}$ und $4^{\circ}$ findet man in [6]. Die Bedingung $3^{\circ}$ ist eine Folge der Irreduzibilität.

Wendet man nun $1^{\circ}$ und $2^{\circ}$ auf die vorliegende Gleichung an, so erhält man

$$
\begin{gathered}
L(z, 1, y) \equiv 1, \\
M(z, 1, y) \equiv \sum_{j=0}^{2} a_{j}(z) y^{j}
\end{gathered}
$$

und

$$
N(z, 1, y) \equiv \sum_{j=0}^{4} b_{j}(z) y^{j}
$$


Ist $h$ der Homogenitätsgrad von $\Omega$ und sind damit $h-2, h-1$ und $h$ die Grade der Polynome $L, M$ und $N$, so folgt

$$
\begin{gathered}
L\left(z, w, w_{1}\right) \equiv w^{h-2}, \\
M\left(z, w, w_{1}\right) \equiv w^{h-3} \sum_{j=0}^{2} a_{j}(z) w^{2-j} w_{1}^{j} \equiv w^{h-4} w P\left(z, w, w_{1}\right)
\end{gathered}
$$

und

$$
N\left(z, w, w_{1}\right) \equiv w^{h-4} \sum_{j=0}^{4} b_{j}(z) w^{4-j} w_{1}^{j} \equiv w^{h-4} Q\left(z, w, w_{1}\right) .
$$

Damit ist

$$
\Omega\left(z, w, w_{1}, w_{2}\right)=w^{h-4}\left(\left(w w_{2}\right)^{2}+2 P\left(z, w, w_{1}\right) w w_{2}+Q\left(z, w, w_{1}\right)\right)
$$

wie behauptet. Denn wegen der Irreduzibilität von $\Omega$ ist $h=4$ bzw. $h=3$ (falls $\left.b_{4} \equiv 0\right)$ bzw. $h=2 \quad$ (falls $b_{4} \equiv b_{3} \equiv a_{2} \equiv 0$ ).

Zum Beweis von Satz 2 kann man von (3) ausgehen, so da $y=w^{\prime} / w$ der Gleichung (16) genügt. Für $c(z) \equiv-1$ ist jede Lösung von (16) eine ganze Funktion, und $w(z)$ hat die in Satz 2 (a) angegebene Gestalt. Ist hingegen $c(z) \not \equiv-1$, so hat nach Wittich [8] jede transzendente Lösung von (16) unendlich viele einfache Polstellen $z_{1}, z_{2}, \ldots$ Die Residuen $R_{j}=\operatorname{Res}_{z_{j}} y$ müssen ganze Zahlen sein, woraus $-R_{j}+\left(1+c\left(z_{j}\right)\right) R_{j}^{2}=0$ oder $\left|1+c\left(z_{j}\right)\right|=1 /\left|R_{j}\right| \leqq 1$ folgt. Damit ist $c(z)+1 \equiv 1 / n$, $n \neq 0$ eine ganze Zahl. Die Funktion $w$ besitzt nur $n$-fache Nullstellen und keine Pole, falls $n>0$ ist, und nur $(-n)$-fache Pole und keine Nullstellen für $n<0$. Daher ist $w=v^{n}$ mit einer ganzen Funktion $v$, die, wie man leicht nachrechnet, der in Satz 2 (b) angegebenen linearen Differentialgleichung genügt. Damit ist Satz 2 vollständig bewiesen.

\section{Beweis der Sätze 4 und 5}

Wieder wird die Substitution $w^{\prime} / w=y$ verwendet. Sie transformiert (4) in

$$
\begin{gathered}
y^{\prime 2}+2\left(a_{0}+a_{1} y+\left(a_{2}+1\right) y^{2}\right) y^{\prime}+b_{0}+b_{1} y \\
+\left(b_{2}+2 a_{0}\right) y^{2}+\left(b_{3}+2 a_{1}\right) y^{3}+\left(b_{4}+2 a_{2}+1\right) y^{4}=0,
\end{gathered}
$$

oder, anders geschrieben, in

$$
\left[y^{\prime}+a_{0}+a_{1} y+\left(a_{2}+1\right) y^{2}\right]^{2}=D(z, y),
$$

wobei $D(z, y)$ durch (7) gegeben ist. Nach Voraussetzung besitzt (17) eine transzendente meromorphe Lösung mit nur endlich vielen Polstellen. Dies bedingt

$$
b_{4}(z)+2 a_{2}(z)+1 \equiv 0 .
$$

Denn wäre dies nicht der Fall, so könnte man (17) in der Form

$$
y^{4}=T_{3}\left(z, y, y^{\prime}\right)
$$


mit einem Polynom $T_{3}$ in $y$ und $y^{\prime}$ vom Grad 3 schreiben und mit Hilfe eines bekannten Lemmas von Clunie [1] auf $m(r, y)=S(r, y)$ schließen, was zusammen mit $N(r, y)=O(\log r)$ zu einem Widerspruch führte. Mit einer ähnlichen Begründung folgt

$$
b_{3}(z)+2 a_{1}(z) \equiv a_{2}(z)+1 \equiv 0 .
$$

Andernfalls folgte zusammen mit (18) aus (17)

$$
y^{2}\left[\left(b_{3}+2 a_{1}\right) y+2\left(a_{2}+1\right) y^{\prime}\right]=T_{2}\left(z, y, y^{\prime}\right)
$$

mit einem Polynom $T_{2}$ in $y$ und $y^{\prime}$ von Grad 2. Mit

$$
c_{0}(z):=\left(b_{3}(z)+2 a_{1}(z)\right) y(z)+2\left(a_{2}(z)+1\right) y^{\prime}(z)
$$

erhielte man wiederum nach dem Clunieschen Lemma $m\left(r, c_{0}\right)=S(r, y)$ und zusammen mit $N\left(r, c_{0}\right) \leqq 2 N(r, y)=O(\log r)$ schießlich

$$
T\left(r, c_{0}\right)=S(r, y) .
$$

Somit ist $y$ Lösung einer linearen Differentialgleichung $y^{\prime}=\alpha(z)+\beta(z) y$ mit Koeffizienten $\alpha$ und $\beta$, die der Bedingung $T(r, \alpha)+T(r, \beta)=S(r, y)$ genügen. Wird dies in (17) eingetragen, so erhält man $D(z, y)=\left[\alpha(z)+a_{0}(z)+\left(a_{1}(z)+\beta(z)\right) y+\left(a_{2}(z)+1\right) y^{2}\right]^{2}$, und diese Bedingung steht im Widerspruch zur Irreduzibilität der betrachteten Gleichung (vgl. [6]).

Aus (18) und (19) folgt $a_{2}(z) \equiv-1$ und $b_{4}(z) \equiv 1$.

Nach dem bisher Gezeigten ist die Diskriminante $D(z, y)$ höchstens quadratisch in $y$. Nach den Aussagen $3^{\circ}$ und $4^{\circ}$ des Hilfssatzes aus Nummer 5 ist

oder

$$
D(z, y)=c(z)\left(y-\varrho_{1}(z)\right)
$$

$$
D(z, y)=c(z)\left(y-\varrho_{1}(z)\right)\left(y-\varrho_{2}(z)\right), \quad \varrho_{1}(z) \not \equiv \varrho_{2}(z),
$$

wobei $\varrho_{1}$ und $\varrho_{2}$ der (in diesem Fall linearen) Differentialgleichung

$$
\varrho^{\prime}+a_{0}(z)+a_{1}(z) \varrho=0
$$

genügen. Jede Lösung von (22) ist aber eine ganze Funktion. Somit sind die bisher nur als algebraisch bekannten Funktionen $\varrho_{1}$ und $\varrho_{2}$ Polynome. Aussage (a) von Satz 4 ist mit $p \equiv \varrho_{1}$ bereits bewiesen. Zum Beweis von (b) ist zu bemerken, daß (22) nur für $a_{1}(z) \equiv 0$ zwei verschiedene Polynomlösungen besitzen kann. Weiter besitzt nach dem Eindeutigkeitssatz für Differentialgleichungen erster Ordnung das Polynom $\varrho_{1}-\varrho_{2}$ keine Nullstellen und ist daher konstant, d. h. es gilt $\varrho_{1}=p+\chi, \varrho_{2}=p-\varkappa$ mit einem Polynom $p, p^{\prime} \equiv-a_{0}$, und einer Konstanten $x \neq 0$. Dies beweist Teil (b) von Satz 4.

Zum Beweis von Satz 5 ist zu zeigen, daß jede Lösung von

$$
\left[v^{\prime}+a_{1}(z) v\right]^{2}=\gamma^{2}(z) v
$$

bzw.

$$
v^{\prime 2}=\gamma^{2}(z)\left(v^{2}-x^{2}\right)
$$


eine ganze Funktion ist. Dies bestätigt man im zweiten Fall explizit: $v(z)=\chi \sin g(z)$, $g^{\prime}(z)=i \gamma(z)$, und im ersten Fall mit Hilfe der Substitution $v=T^{2}$, die auf die lineare Differentialgleichung

führt.

$$
2 T^{\prime}+a_{1}(z) T=\gamma(z)
$$

\section{Beweis der Sätze 6 und 7}

Aus der Residuenbedingung $(R)$ folgt zunächst, daß $a_{2}$ und $b_{4}$ konstant sind. Dies ist unmittelbar klar für $b_{4}+2 a_{2}+1 \equiv 0$, in welchem Fall $2\left|1+a_{2}(z)\right|=1 /|R| \leqq 1$ und hieraus die Konstanz von $a_{2}$ und $b_{4}$ folgt. Ist $b_{4}+2 a_{2}+1 \neq 0$, so seien, bei festem $z, n$ und $m$ die ganzzahligen Lösungen von (11). Es ist dann $\left|1+2 a_{2}+b_{4}\right|=1 /|n m| \leqq 1$ und somit $1+2 a_{2}+b_{4}$ konstant, etwa $=\lambda$. Das Produkt $n m$ ist dann unabhängig von $z$ gleich $1 / \lambda$, woraus insbesondere die Beschränktheit von $n$ und $m$ (in Abhängigkeit von $z$ ) folgt. Damit ist auch $2\left|1+a_{2}\right|=|\lambda||n+m|$ beschränkt und $a_{2}$ konstant.

Zunächst wird der Fall $b_{4} \equiv a_{2}^{2} \quad$ (d. h. $a_{2} \equiv(1-n) / n$ und $\left.b_{4}=((n-1) / n)^{2}\right)$ behandelt. Für $y=w^{\prime} / w$ erhält man in der üblichen Weise

$$
\left[y^{\prime}+a_{0}+a_{1} y+\frac{1}{n} y^{2}\right]^{2}=D(z, y)
$$

wobei $D(z, y)$ durch (7) gegeben ist. In einer Polstelle von $y$, von denen es nach Voraussetzung unendlich viele gibt, hat die linke Seite von (25) einen Pol von gerader Ordnung. Daher ist der Grad von $D(z, y)$ in bezug auf $y$ eine gerade Zahl und wegen $a_{2}^{2}-b_{4}$ (=führender Koeffizient von $\left.D(z, y)\right)=0$ und Aussage $3^{\circ}$ des Hilfssatzes aus Nummer 5 ist

$$
D(z, y)=c_{0}(z),\left(y-\varrho_{1}(z)\right)\left(y-\varrho_{2}(z)\right),
$$

wobei $\varrho_{1}$ und $\varrho_{2}, \varrho_{1}(z) \not \equiv \varrho_{2}(z)$, nach dem eben erwähnten Hilfssatz der Riccatischen Differentialgleichung

$$
\varrho^{\prime}+a_{0}+a_{1} \varrho+\frac{1}{n} \varrho^{2}=0
$$

genügen. Insbesondere sind $\varrho_{1}$ und $\varrho_{2}$ als Lösungen von (27) eindeutige rationale Funktionen.

Zunächst werde angenommen, da $\beta \varrho_{1}$ und $\varrho_{2}$ Polynome sind. Dann ist nach dem Eindeutigkeitssatz für (27) $\varrho_{1}-\varrho_{2}$ stets von Null verschieden, also $\varrho_{1}=p+\varkappa, \varrho_{2}=p-\varkappa$ mit einem Polynom $p$ und einer komplexen Zahl $\varkappa \neq 0$. Dies bedeutet aber für (27) $a_{1}(z)=(-2 / n) p(z)$ und $a_{0}(z)=(1 / n)\left(p^{2}(z)-x^{2}\right)-p^{\prime}(z)$. Wird dies in (25) eingesetzt und die Substitution $w^{\prime} / w=y$ rückgängig gemacht, so erhält man die Gleichung (a) aus Satz 6 mit $c(z):=c_{0}(z)$.

Nun sei etwa $\varrho_{1}$ kein Polynom. Aus dem Eindeutigkeitssatz für (27) folgt dann $\varrho_{1}-\varrho_{2}=1 / Q, Q$ ein Polynom. Weiter folgt aus (27), da $\varrho_{1}$ und $\varrho_{2}$ nur einfache Pole mit Residuen $n$ besitzen (die Gleichung für die Residuen $r$ von $\varrho$ ist $-r+(1 / n) r^{2}=0$ ). 
Die Funktionen $\varrho_{1}$ und $\varrho_{2}$ können keine gemeinsame Polstelle besitzen, wie aus dem Eindeutigkeitssatz für die Gleichung $\sigma^{\prime}-1 / n-a_{1} \sigma-a_{0} \sigma^{2}=0$ folgt, die man aus (27) durch Stürzung $\sigma=1 / \varrho$ erhält. Zusammen ergibt dies $\varrho_{j}=n Q_{j}^{\prime} / Q_{j}+p_{j}$ mit Polynomen $p_{j}$ und $Q_{j}, Q_{1} \cdot Q_{2}=Q$. Aus $\varrho_{1}-\varrho_{2}=n\left(Q_{1}^{\prime} / Q_{1}-Q_{2}^{\prime} / Q_{2}\right)+p_{1}-p_{2}=1 / Q$ folgt dann $p_{1} \equiv p_{2}=: p$. Setzt man $\varrho_{1}=n Q_{1}^{\prime} / Q_{1}+p$ bzw. $\varrho_{2}=n Q_{2}^{\prime} / Q_{2}+p$ in Gleichung (27) ein, so erhält man für $t=Q_{1}, Q_{2}$ die lineare Differentialgleichung

$$
t^{\prime \prime}+\left(a_{1}(z)+\frac{2}{n} p(z)\right) t^{\prime}+\frac{1}{n}\left(p^{\prime}(z)+a_{0}(z)+a_{1}(z) p(z)+\frac{p^{2}(z)}{n}\right) t=0 .
$$

Diese besitzt nach Konstruktion ein Fundamentalsystem, bestehend aus den Polynomen $Q_{1}$ und $Q_{2}$, woraus

$$
a_{1}+\frac{2}{n} p \equiv 0
$$

(die Wronskideterminante ist konstant) und $p^{\prime}+a_{0}+a_{1} p+p^{2} / n=-n Q_{1}^{\prime \prime} / Q_{1} \rightarrow 0(z \rightarrow \infty)$, also

$$
p^{\prime}+a_{0}+a_{1} p+\frac{p^{2}}{n} \equiv 0
$$

folgt. Damit ist $Q_{j}^{\prime \prime} \equiv 0$, also $Q_{1}(z)=c_{1}\left(z-z_{1}\right)$ linear $\left(\varrho_{1}\right.$ besitzt Polstellen), und $Q_{2}(z)=c_{2}$ konstant oder linear, $Q_{2}(z)=c_{2}\left(z-z_{2}\right)$. Man erhält die Gleichungen (b) bzw. (c) mit $c(z):=c_{0}(z) /\left(z-z_{1}\right)$ bzw. $c(z):=c_{0}(z) /\left(z-z_{1}\right)\left(z-z_{2}\right)$.

Nunmehr sei $b_{4} \neq a_{2}^{2}$. Dann hat $D(z, y)$ bezüglich $y$ den Grad 4 und, da der führende Koeffizient $a_{2}^{2}-b_{4}$ von $D(z, y)$ konstant ist, besitzen die Lösungen $y=\varrho(z)$ von $D(z, y)=0$ im endlichen keine eigentlichen oder algebraischen Polstellen. Nach dem Hilfssatz aus Nummer 5 sind nur die folgenden Fälle möglich:

(i) vier verschiedene Lösungen $\varrho_{1}, \varrho_{2}, \varrho_{3}, \varrho_{4}$;

(ii) zwei oder drei verschiedene Lösungen $\varrho_{1}, \varrho_{2}, \sigma, \sigma$.

Dabei ist $\sigma \equiv \varrho_{2}$ im zweiten Fall zugelassen, was einer dreifachen Lösung entspricht. In jedem Fall sind die $\varrho_{j}$ Lösungen der Riccatischen Differentialgleichung

$$
\varrho^{\prime}+a_{0}+a_{1} \varrho+\left(a_{2}+1\right) \varrho^{2}=0
$$

und damit Polynome. Letzteres gilt auch für $\sigma$. Nach dem Eindeutigkeitssatz für (31) sind die Differenzen $\varrho_{i}-\varrho_{j}$ konstant und für $i \neq j$ von Null verschieden.

Im Falle vier verschiedener Lösungen von (31) ist $\varrho_{j}=p+\chi_{j}, p$ ein Polynom und $\varkappa_{i} \neq \varkappa_{j}$ für $i \neq j$. Es ist leicht zu sehen, daß dies nur für $a_{1} \equiv a_{2}+1 \equiv 0$ und $p^{\prime}+a_{0} \equiv 0$ möglich ist. Mit $a_{2}=-1$ folgt schließlich aus der Residuenbedingung $(R)$

$$
1+\left(b_{4}-1\right) R^{2}=0,
$$

also $a_{2}^{2}-b_{4}=1 / n^{2}$. Insgesamt erhält man so Gleichung (d). Es bleibt der Fall (ii) zu behandeln. Wie oben findet man $\varrho_{1}=p+\varkappa$ und $\varrho_{2}=p-\varkappa$ mit einem Polynom 
und einer Konstanten $\varkappa \neq 0$, wobei

$$
p^{\prime}+a_{0}+a_{1}(p \pm \varkappa)+\left(1+a_{2}\right)(p \pm \varkappa)^{2}=0
$$

erfüllt sein muß. Die Differentialgleichung für $y=w^{\prime} / w$ lautet in diesem Fall

$$
\left[y^{\prime}-p^{\prime}+\left(1+a_{2}\right)\left((y-p)^{2}-x^{2}\right)\right]^{2}=\left(a_{2}^{2}-b_{4}\right)\left((y-p)^{2}-x^{2}\right)(y-p-s)^{2},
$$

wobei $p+s=\sigma$ gesetzt ist.

Aus der Residuenbedingung $(R)$ folgt schließlich $1+a_{2}=1 / 2 n$ und $a_{2}^{2}-b_{4}=$ $1 /\left(4 n^{2}\right)$ (das entspricht Gleichung (e)) oder $1+a_{2}=(n+m) /(2 n m)$ und $a_{2}^{2}-b_{4}=$ $((n-m) /(2 n m))^{2} \neq 0$, entsprechend Gleichung (f). Damit ist Satz 6 vollständig bewiesen.

Zum Beweis von Satz 7 werden die Gleichungen (a)-(f) durch passende Substitutionen in Gleichungen übergeführt, von denen bekannt ist, daß ihre Lösungen in $C$ meromorph sind. Es sind dies der Reihe nach die Substitutionen

$$
\begin{aligned}
& \frac{w^{\prime}}{w}-p=n v, \quad \frac{v-\lambda}{v+\lambda}=T^{2}, \quad \varkappa=n \lambda ; \\
& \frac{w^{\prime}}{w}-p=n v, \quad \frac{v}{\left(z-z_{1}\right) v-1}=T^{2} \\
& \frac{w^{\prime}}{w}-p=n v, \quad \frac{\left(z-z_{2}\right) v-1}{\left(z-z_{1}\right) v-1}=T^{2} \text {; } \\
& \frac{w^{\prime}}{w}-p=n v, \quad \varkappa_{j}=n \lambda_{j} \\
& \frac{w^{\prime}}{w}-p=n v, \quad \frac{v-\lambda}{v+\lambda}=T^{2}, \quad \varkappa=n i, \quad s=n q ; \\
& \frac{w^{\prime}}{w}-p=v, \quad \frac{v-\varkappa}{v+\varkappa}=T^{2} .
\end{aligned}
$$

Sie führen auf folgende Differentialgleichungen:

$$
\begin{gathered}
2 T^{\prime}+2 \lambda T+\gamma(z)\left(T^{2}-1\right)=0 ; \\
2 T^{\prime}-\gamma(z)\left(1-\left(z-z_{1}\right) T^{2}\right)=0 ; \\
2 T^{\prime}-\gamma(z)\left(z-z_{2}-\left(z-z_{1}\right) T^{2}\right)=0 ; \\
v^{\prime 2}=\left(v-\lambda_{1}\right)\left(v-\lambda_{2}\right)\left(v-\lambda_{3}\right)\left(v-\lambda_{4}\right) ; \\
4 T^{\prime}+2 \lambda T+\left(q(z)-\lambda_{-}-(q(z)+\lambda) T^{2}\right)=0 ; \\
4 T^{\prime}+2 x \frac{n+m}{n m} T+\frac{n-m}{n m}\left(s(z)-\varkappa-(s(z)+\chi) T^{2}\right)=0 .
\end{gathered}
$$

Durch die verwendeten Substitutionen sind die im Anschluß an Satz 7 erwähnten speziellen Lösungen weggefallen (sie entsprechen den Funktionen $v \equiv \pm \lambda$ bei (a') 
und $\left(\mathrm{e}^{\prime}\right), v \equiv \pm \varkappa$ bei $\left(\mathrm{f}^{\prime}\right), v \equiv 0$ bei ( $\left.\mathrm{b}^{\prime}\right)$ bzw. $v \equiv 1 /\left(z-z_{j}\right)$ bei $\left(\mathrm{b}^{\prime}\right)$ und $\left(\mathrm{c}^{\prime}\right)$ und $v \equiv \lambda_{j}$ bei $\left(d^{\prime}\right)$.

Die verbleibenden Funktionen sind bei ( $\left.d^{\prime \prime}\right)$ doppeltperiodisch (vgl. [5]) und in den restlichen Fällen als Lösungen Riccatischer Differentialgleichungen in $C$ meromorph. Die Residuenbedingung $(R)$ garantiert, daß $v$ nur einfache Polstellen mit ganzzahligen Residuen besitzt und daher eine logarithmische Ableitung ist. Dies zeigt, daß mit $v$ auch stets $w$ in $C$ meromorph ist und beweist Satz 7 .

Daß tatsächlich unendlich viele Null- oder Polstellen auftreten, sei am Fall (f) demonstriert (die Beweise in den restlichen Fällen verlaufen analog). Es genügt zu zeigen, daß $v$ unendlich viele Polstellen hat, oder, was dasselbe ist, daß $T$ einen der Werte +1 und -1 (oder beide) unendlich oft annimmt. Nun ist aber weder $T(z) \equiv 1$ noch $T(z) \equiv-1$ Lösung von (f"), so daß nach [8] $T$ jeden der Werte +1 und -1 unendlich oft annimmt.

\section{Literatur}

[1] Clunie, J.: On integral and meromorphic functions. - J. London Math. Soc. 37, 1962, 17- 27.

[2] Hayman, W. K.: Meromorphic functions. - Clarendon Press, Oxford, 1964.

[3] Malmquist, J.: Sur les fonctions à un nombre fini de branches définies par les équations différentielles du premier ordre. - Acta Math. 36, 1913, 297-343.

[4] Nevantinna, R.: Eindeutige analytische Funktionen. - Die Grundlehren der mathematischen Wissenschaften 46, Verlag von Julius Springer, Berlin, 1936.

[5] Rellich, F.: Elliptische Funktionen und die ganzen Lösungen von $y^{\prime \prime}=f(y)$. - Math. Z. 47, 1940, 153-160.

[6] Steinmetz, N.: Ein Malmquistscher Satz für algebraische Differentialgleichungen erster Ordnung. - J. Reine Angew. Math. 316, 1980, 44-53.

[7] Steinmetz, N.: Über das Anwachsen der Lösungen homogener algebraischer Differentialgleichungen zweiter Ordnung. - Manuscripta Math. 32, 1980, 303-308.

[8] Wrtrich, H.: Einige Eigenschaften der Lösungen von $w^{\prime}=a(z)+b(z) w-c(z) w^{2}$. - Arch. Math. (Basel) 5, 1954, 226-232.

Universität Karlsruhe

Mathematisches Institut I

Postfach 6380

D 7500 Karlsruhe

Deutschland

Eingegangen am 22. Oktober 1981 